\title{
The Effect of the Ferrofluid Magnetic Particle Concentration on the Thermal Diffusivity
}

\author{
S.M. Shibli*(a) ${ }^{*}$ A.L.L. Dantas ${ }^{(a)}$, and A. Bee ${ }^{(b)}$ \\ (a)Instituto de Física, Universidade de São Paulo, \\ Caixa Postal 66318, 05315-970, São Paulo, SP, BRAZIL (b)LI2C Colloides Inorganic UMR 7612, \\ Universite Pierre et Marie Curie, Case 63, 4 place Jussieu, \\ 75252 Paris - Cedex-05, France
}

Received on 20 December, 2000

\begin{abstract}
Thermal diffusivity results obtained with the collinear mirage technique, are reported for different concentrations surfacted ferrofluid and for a set of acid ferrofluids with variable $\mathrm{pH}$ values. The collinear mirage technique was used because the materials are semitransparent and have low thermal diffusivities. The results show that, as the ferrofluid magnetic particles concentration increases, the thermal diffusivity also increases. Their behaviour differs from that reported for ferronematics [1]. The different geometry of thermal waves propagating through the material can account for the difference.
\end{abstract}

\section{Introduction}

A ferrofluid is a colloidal suspension of ferrimagnetic nanoparticles dispersed in a carrier solvent. It has the fluidity of a homogeneous isotropic liquid and a high magnetic susceptibility. These two characteristics have led to numerous technical applications, [2] - [4]. Optical [5], magnetic [6] and structural [7] properties, among others, have been studied. However, to our knowledge, only one report on thermal properties, mainly the thermal diffusivity can be found in the literature [8].

The thermal diffusivity was obtained with the collinear mirage technique. The collinear configuration provides a reliable photothermal alternative for measuring thermal diffusivities of semitransparent materials. [9] This technique was also chosen due to its well known potential for avoiding large errors from experimental parameters, and also to its high sensitivity to the thermal properties of the sample. Another important advantage is that, due to the existence of simple linear relations between two measurable parameters, the resulting slope can be used to obtain the thermal diffusivity directly.

The collinear mirage effect is produced by the absorption of a modulated exciting beam which heats the medium, generating a refractive index gradient within the sample. A second (probe) laser beam runs, through the sample, collinearly and in a direction opposite to that of the pump beam. The probe beam is deflected by the gradient in the index of refraction due to the thermal gradients generated by the exciting beam. The magnitude of the beam deflection, known as mirage deflection, carries information about the thermal properties of the sample.

We have used the collinear mirage technique to measure the thermal diffusivity of ferrofluids. We have used the linear relation between the phase of the collinear mirage deflection and the spacing between the exciting and probe beams, for a fixed frequency to obtain the diffusivity. This model is known as the phase method, [10]-[12] and is indicated for analysing data obtained in collinear mirage geometries.

In Section II this model is outlined, and its validity and limitations for the calculation of the collinear deflection are discussed. The experimental details are described in Sec.III. The data are analysed in Sec.IV. Finally, in Sec.V we present the conclusions and compare the results to those reported for ferronematics, which are lyotropic nematic liquid crystals doped with small amounts of surfacted ferrofluids. This material was measured with the same photothermal used here and had been analysed in the same way.

\section{Theory}

The theory for the phase angle between probe and exciting beams follows Walton [13] and is summarized below:

\footnotetext{
*(a) Author to whom all correspondence should be addressed:shibli@if.usp.br
} 
Assume that the angle between the exciting and probe beams is sufficiently small that the two may be assumed to be parallel: with standard results from Carslaw and Jaeger [14] the temperature, $T(r, t)$ at a point a distance $r$ from the center of the exciting beam is, with $\alpha$ as the thermal diffusivity, and with a gaussian profile of radius $a, e^{-\left(\frac{r}{a}\right)^{2}}$, for the exciting beam,

$$
T(r, t)=A e^{i \omega t} K_{0}\left(r \sqrt{i} \sqrt{\frac{\omega}{\alpha}}\right) \int_{0}^{r} d r^{\prime} e^{-\left(\frac{r^{\prime}}{a}\right)^{2}} I_{0}\left(r^{\prime} \sqrt{\frac{\omega}{\alpha}} \sqrt{i}\right)
$$

$K_{o}$ and $I_{0}$ are Bessel functions. A is a constant that depends on geometrical factors, detector sensitivity, absorptivity of the medium, etc. If the exciting beam is narrow, i.e. if $a \sqrt{\frac{\omega}{\alpha}}<1, I_{0}\left(r^{\prime} \sqrt{\frac{i \omega}{\alpha}}\right) \simeq 1$, and

$$
T(r, t) \simeq A e^{i \omega t} K_{0}\left(r \sqrt{i} \sqrt{\frac{\omega}{\alpha}}\right) \operatorname{erf}\left(\frac{r}{a}\right)
$$$$
\text { If } r>a, \operatorname{erf}\left(\frac{r}{a}\right) \rightarrow 1, \text { and } T(r, t) \simeq
$$
$A e^{i \omega t} K_{0}\left(r \sqrt{i} \sqrt{\frac{\omega}{\alpha}}\right)$

Now, $K_{0}(z \sqrt{i})=\operatorname{ker}(z)+i k e i(z)=N_{0}(z) e^{i \psi(z)}$ where $N_{0}=\sqrt{k e r^{2} z+k e i^{2} z}$. Thus with $\psi=$ $\tan ^{-1} \frac{\operatorname{kei}(z)}{\operatorname{ker}(z)}$,

$$
T(r, t) \simeq A e^{i\left[\omega t+\psi\left(r \sqrt{\frac{\omega}{\alpha}}\right)\right]} N_{0}\left(r \sqrt{\frac{\omega}{\alpha}}\right)
$$

$\psi\left(r \sqrt{\frac{\omega}{\alpha}}\right)$ is the phase shift in the temperature, and will also be the phase shift in the detected beam which is proportional to $\frac{\partial T}{\partial r}$. An asymptotic expansion for $\psi\left(r \sqrt{\frac{\omega}{\alpha}}\right)$ yields

$\left.\psi\left(r \sqrt{\frac{\omega}{\alpha}}\right)=-\frac{\pi}{8}-r \sqrt{\frac{\omega}{2 \alpha}}\right)+\frac{1}{8 r} \sqrt{\frac{\alpha}{2 \omega}}-\ldots$

Thus a plot of the phase against the separation of the two beams will yield a straight line in the region where $r \sqrt{\frac{\omega}{\alpha}}>1$, whose slope is $\sqrt{\frac{\omega}{2 \alpha}}$, and from which the diffusivity $\alpha$ may be deduced. This result agrees with numerical solutions obtained by Salazar et al. [10][12].

We have reported that for anisotropic complex fluids, as ferronematics, these linear relations also apply, but not in a direct way. [1] Here, for this point of view, we have a simpler case, since it is known that ferrofluids is an isotropic material, in the absence of a magnetic field, which is the condition kept throughout this work. So, firstly we know that the material analyzed here is homogeneous and isotropic. Secondly, we assume that the geometrical configuration used extends to an infinite medium.

\section{Experimental}

\section{III.1 Surfacted Ferrofluids}

This ferrofluid, acquired from Ferrofluidics Co., consists of magnetic particles, with typical dimensions of about $10 \mathrm{~nm}$. [3] Each particle is coated with a surfactant agent to prevent their aggregation. The nanoparticles are magnetic monodomains of magnetite
$\left(\mathrm{Fe}_{3} \mathrm{O}_{4}\right)$, dispersed in water at a concentration of about $1.43 \times 10^{16}$ grains $/ \mathrm{cm}^{3}$ in the concentrated solution. Different concentrations of this ferrofluid were used, varying the $[\mathrm{Fe}]$ from 0.03 to $0.05 \mathrm{~mol} / \mathrm{L}$, in water.

\section{III.2 Acid Ferrofluids}

The acid magnetic fluid used is an aqueous ferrofluid. The suspended nanoparticles are magnetic monodomains of maghemite $\left(\gamma-\mathrm{Fe}_{2} \mathrm{O}_{3}\right)$, synthesized through Massart's method [15], provided by Colloides Inorganic Group at Universite Pierre et Marie Curie. The colloidal stability of the solution is ensured by a screened electrostatic repulsion between particles. The $\mathrm{pH}$ value of the medium controls the superficial density of charges of the magnetic particles and thus the stability of the solutions. The system evolves by increase of the $\mathrm{pH}$ from a soluble state (acid ferrofluid) towards floculation $(\mathrm{pH}=5)$ close to the point of zero charge located at $\mathrm{pH}$ about 7.3 for maghemite. Nevertheless, the samples measured here are in a $\mathrm{pH}$ range in which the ferroparticles remain stable, ensuring the global stability of the solution.

The samples described above were encapsulated in cuvettes $0.5 \mathrm{~cm}$ thick on the side and $1.0 \mathrm{~cm}$ wide, which allowed us to consider the sample as an infinite medium. The measurements were performed at room temperature $\left(T=22^{\circ} C\right)$, and in the earth's magnetic field, which is essentially negligible. The collinear mirage set-up is shown in figure 1. [16]

The Mirage deflection, probed by the probe beam, is due to the change of the index of refraction at the sample. This deflection is detected by a position sensitive detector. The curve obtained from plotting the amplitude of the mirage signal as a function of the beam separation (Fig. 2) was used for calibrating the alignment of our set up. Thus, to determine the reliability of each measurement, first of all we have measured the mirage signal, which is obtained directly in Volts, from the amplitude values of the lock-in. The signal was then plotted against the beams separation, in micrometers, for each frequency, as seen in Fig. 2.

We have also measured the time dependence effect in our photothermal measurements. From this analysis, we have confirmed that no significant variation is observed in our data, i.e., no particle diffusion and thermally-induced variation in particle concentration, such as that reported by Du and Luo [8], was observed in our measurements. The absence of temporal dependence of the present photothermal measurements was checked during a period of 90 minutes. 


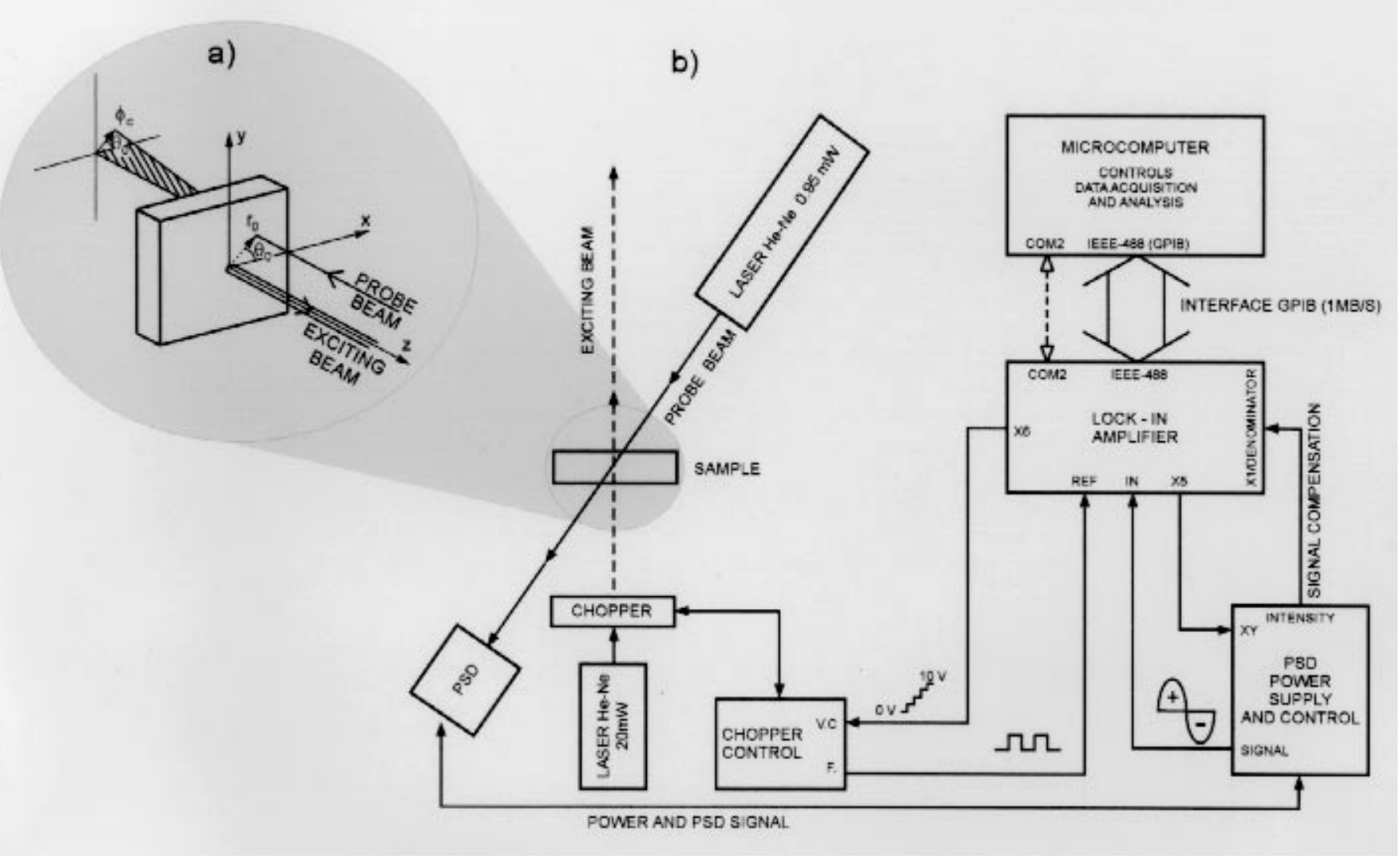

Figure 1. a)Representation of the geometry for the collinear mirage deflection $\phi_{C}$. The probe beam is separated by a distance $r_{0}$ and oriented by an angle $\theta_{0}$ from the exciting beam which is located at coordinates $\left.(0,0) ; \mathrm{b}\right)$ Schema of the experimental setup used in our collinear mirage technique.

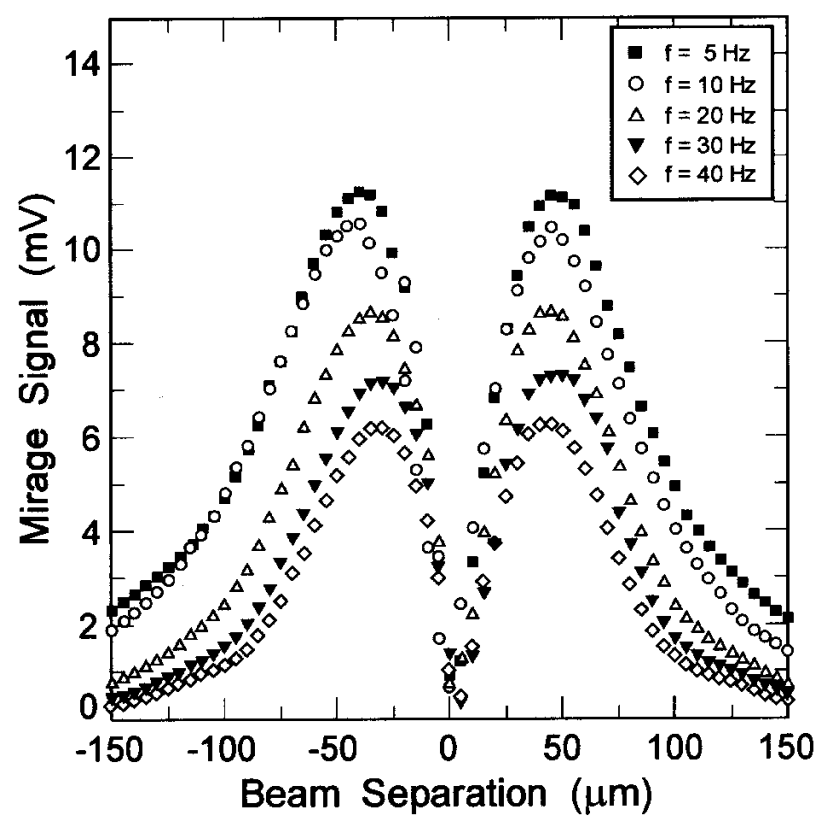

Figure 2. The graph of the mirage signal as a function of the separation between the pump and probe beams, of the variable frequencies (f) for the raw data of the ferrofluid sample with the highest ferrofluid concentration. The alignment observed for both peaks at each frequency insures the reliability of the measurements and the calibration of the setup.

\section{Results and Discussion}

Fig. 3 shows the experimental data for a sample with surfacted ferrofluid concentration of approximately $0.05 \mathrm{~mol} / L$, for frequencies values between 5 and $40 \mathrm{~Hz}$. This highest doped sample shows the most reliable data throughout the frequency range. The linearity between beam separation and phase only holds for a limited range of $\mathrm{r}: r \sqrt{\frac{\omega}{\alpha}}$ must be larger than 1 , which provides a lower limit for r. An upper limit exists due to the decrease in signal strength as $r$ increases. Note that the best linearity is obtained, as would be expected, for the two lowest frequencies. A least squares fit to the linear part of these data yields the thermal diffusivity.

Results for samples of surfacted ferrofluid with different concentrations are shown in Fig. 4. These were obtained at a frequency of $10 \mathrm{~Hz}$, which provided the best results. The error deduced from the least squares fit was about $5 \%$. The diffusivity increases with ferrofluid concentration. Thus, these thermal properties variations are directly related to the interparticle distances. 


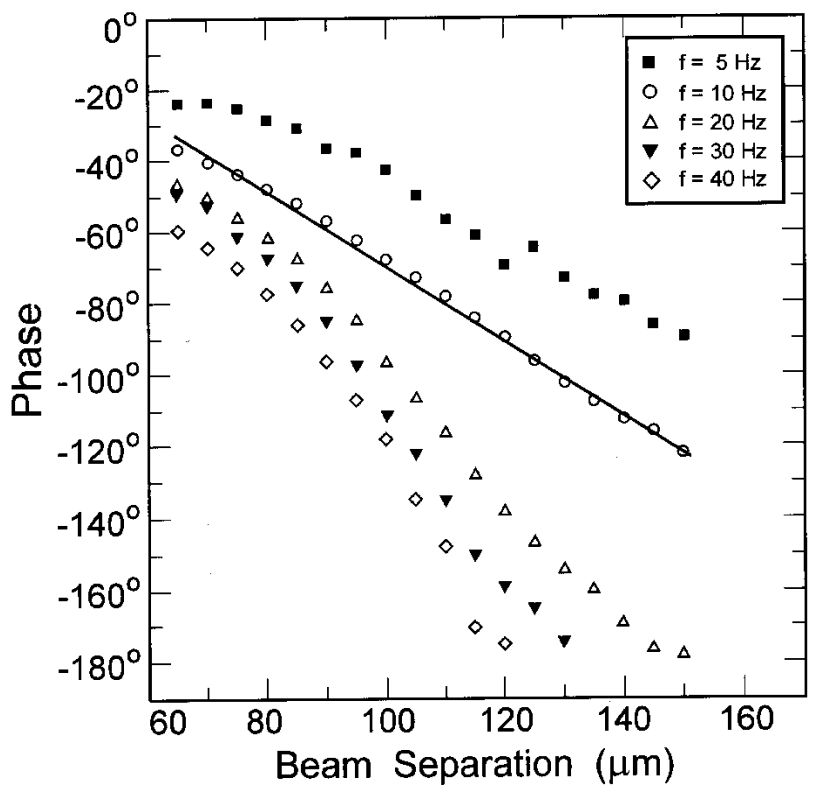

Figure 3. The same data as shown in Fig. 2, for the phase data plotted at the linear part of the beam separation. These data were adjusted until the plotted points coincided with the fitting of a linear slope, used to retrieve the thermal diffusivity. The symbols refer to the same data as that plotted in Fig. 2.

From this experiment two quantities can be deduced. The $\alpha_{S}=1.5 \times 10^{-7} \mathrm{~m}^{2} / \mathrm{s}$ at $[\mathrm{Fe}]=0$ is the pure solvent. The slope of the experimental representation, which is at the first order given by the expression $\alpha_{S}=\frac{K_{\text {part. }} \times \Phi}{\left(K_{\text {part. }}-K_{\text {solvent }}\right)}$, where $\Phi$ is the volume fraction of particles (here $\Phi=1.5 \times 10^{-2}[\mathrm{Fe}]$ ), $K_{\text {part }}$. and $K_{\text {solvent }}$ are the thermal conductivity of the ferrofluid particles and of the solvent, respectively, here $K_{\text {part. }} \approx$ $6.2 W \cdot m^{-1} \cdot K^{-1}$, and $K_{\text {solvent }} \approx 0.6 W \cdot m^{-1} \cdot K^{-1}$, as seen in J. Lenglet thesis [18]. From the theoretical expression of the slope we obtained $1.3 \times 10^{-7}$, and our experimental slope is $1.5 \times 10^{-4}$. The difference between these two results could be explained by the absorption of the solution, since ferrofluids are strong light absorbents.

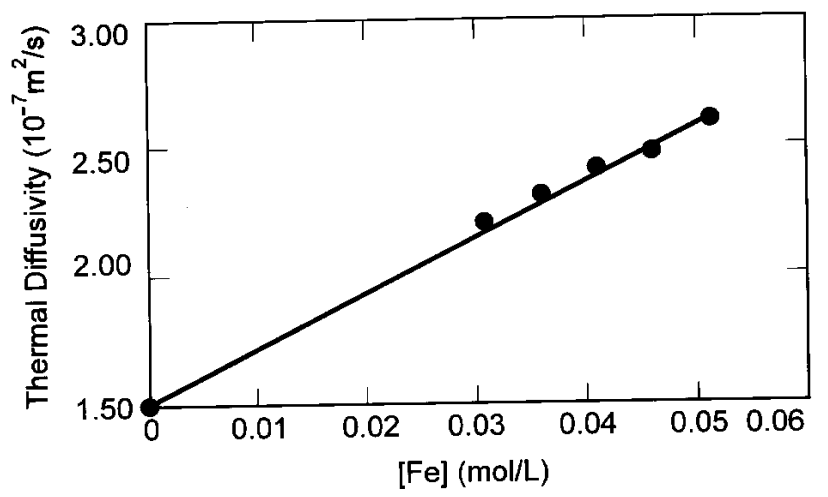

Figure 4. The data of five samples with variable surfacted ferrofluid $(\mathrm{FF})$ concentrations, calculated at frequencies of $10 \mathrm{~Hz}$. From this figure we observe the decrease of the thermal diffusivity with the decrease of FF concentration.
For the set of samples of acid ferrofluid the $\mathrm{pH}$ values used were 2.80 and $3.21,[F e]=0.1 \mathrm{~mol} / \mathrm{L}$ and particles diameter around $7 \mathrm{~nm}$. In the Table 1, we have showed that the thermal diffusivity $(\alpha)$ increases with the increase of $\mathrm{pH}$ values. It has been verified [17] that, a $\mathrm{pH}$ increase induces a surface charge decreasing, consequently the interparticle electrostatic repulsions decrease inducing an aggregation of the ferroparticles. Further studies must be performed for a wider $\mathrm{pH}$ range to obtain an overall picture of the thermal waves behaviour in such ferrofluid samples.

\section{Table 1}

The data of two samples with variable acid ferrofluid (FF) $\mathrm{pH}$, calculated at frequencies of $10 \mathrm{~Hz}$. From this result we observe the increase of the thermal diffusivity with the increase of $\mathrm{FF} \mathrm{pH}$.

\begin{tabular}{ccc}
\hline $\mathrm{pH}$ & 2.80 & 3.21 \\
\hline$\alpha\left(10^{-7} \mathrm{~m}^{2} / \mathrm{s}\right)$ & 3.70 & 4.40 \\
\hline
\end{tabular}

From the results obtained for the two different types of ferrofluid studied so far, we have observed that a close relation exists between the number of ferroparticles and the thermal properties. In despite of the different types of ferrofluids analysed and the large difference in their ferroparticles concentration, the same behaviour was verified, as can be seen by the thermal diffusivity values measured.

\section{Conclusion}

In conclusion, we have obtained thermal diffusivity of samples with two different types of ferrofluids, for several ferroparticles concentrations. The results show that for these ferrofluid samples exists a bound between the thermal properties variation and the interparticle distances. A detailed study about the relation between these two parameters may lead us to some theory from where we'll be able to obtain the interparticle distances by the analysis of the thermal properties of such materials. We have also observed a large gap between the standard theory proposed by J. Lenglet [18] and the experimental results. A tentative proposition of explanation could be that ferrofluid are strong light absorbent, so the absorption coefficient should be taken into account. The details of such a development will be presented in a future paper.

\section{Acknowledgment}

Financial support received from the Brazilian agency Fundação de Amparo à Pesquisa do Estado de São Paulo - FAPESP is gratefully acknowledged. 


\section{References}

[1] S.M. Shibli, A.L.L. Dantas and D. Walton, Appl.Phys.Lett. 72, 674 (1998).

[2] R.E. Rosensweig, Ferrohydrodynamics (Cambridge University Press, Cambridge, England, 1985).

[3] Magnetic Fluids and Applications - Handbook, edited by B. Berkovsky and M. Krakov (Begel-House, New York, 1994).

[4] R. Massart, IEEE Trans.Magn.MAG-17, 1247 (1981).

[5] M.F. da Silva and A.M. Figueiredo Neto, Phys.Rev. E 48, 4483(1993); and references therein.

[6] M.C. Cowley and R.E. Rosensweig, J.Fluid Mech. 30, $671(1967)$.

[7] L.A. do Amaral and F.A.Tourinho, Brazilian J.Phys. 25, 142(1995); and references therein.

[8] T. Du and W. Luo, Appl.Phys.Lett.72, 272 (1998).

[9] A.C. Boccara, D. Fournier and J. Badoz, Appl.Phys.Lett.36,130 (1980).
[10] A. Salazar, A. Sánchez-Lavega and J. Fernández, J.Appl.Phys.74,1539 (1993).

[11] A. Salazar and A. Sánchez-Lavega, Rev. Sci.Instrum. 65,2896 (1994)

[12] A. Salazar, A. Sánchez-Lavega, A. Ocáriz, J. Guitonny, G.C. Pandey, D. Fournier, and A.C. Boccara, J.Appl.Phys.79,3984 (1996).

[13] D. Walton, Appl. Phys. Lett., submitted.

[14] H.S. Carslaw and J.C. Jaeger, "Conduction of heat in solids", (Oxford University, London) (1947).

[15] R. Massart, IEEE Trans.Magn.MAG-17,1247 (1981).

[16] A.L.L. Dantas, D. Walton and S.M. Shibli, Brazilian J. Phys.28, 428 (1998).

[17] E. Hasmonay, A. Bee, J-C Bacri and R. Perzynski, J.Phys. Chem. 103(31), 6421 (2000).

[18] J. Lenglet "Generation de Second Harmonique et Diffusion Rayleigh Forcee dans les Colloides Magnetiques", Thesis Paris-France (1996). 